\title{
Effective Bounds in Euler-Maclaurin-Based Quadrature (Summary for HPCS06)
}

\author{
David H. Bailey \\ Lawrence Berkeley National Lab \\ Berkeley, CA 94720 USA
}

\author{
Jonathan M. Borwein \\ Faculty of Computer Science, Dalhousie University \\ Halifax, NS, B3H 2W5 Canada
}

\begin{abstract}
We analyze the behavior of Euler-Maclaurin-based integration schemes with the intention of deriving accurate and economic estimations of the error. These schemes typically provide very high-precision results (hundreds or thousands of digits), in reasonable run time, even in cases where the integrand function has a blow-up singularity or infinite derivative at an endpoint. Heretofore, researchers using these schemes have relied mostly on ad hoc error estimation schemes to project the estimated error of the present iteration. In this paper, we seek to develop some more rigorous, yet highly usable schemes to estimate these errors.
\end{abstract}

1. Introduction. In the past few years, computation of definite integrals to high precision has emerged as a key tool in experimental mathematics. In particular, it is often possible to recognize an unknown definite integral in analytic terms, provided its numerical value is known to extremely high precision. High precision levels are required because integer relation searches of $n$ terms with $d$-digit coefficients require at least $d n$-digit precision for both input data and relation searching [5, pg. 231]). Such computations often require require highly parallel implementations. The quadrature computation in [3], for instance, required nearly one hour on 1024 processors, and the PSLQ integer relation search in [5, pg. 8] required 44 hours on 32 processors. Moreover, such extreme computations provide excellent tests of HPC systems-for example, one of the present authors identified a difficulty with differing processor speeds on the Virginia Tech system with these calculations.

The tanh-sinh quadrature scheme is the fastest cur- rently known high-precision quadrature scheme, particularly when one counts the time for computing abscissas and weights, although error function quadrature also does well in certain problems [4]. Tanhsinh quadrature has been successfully employed for quadrature calculations up to 20,000-digit precision [3]. It works well for functions with blow-up singularities or infinite derivatives at endpoints [4], and is well-suited for highly parallel implementation [3].

At present, these schemes rely on ad-hoc methods to estimate the error at any given stage. One does not need to rely on these estimates-one can simply continue until two iterations give the same result (except possibly for the last few digits), but this nearly doubles the overall run time, which of course is an issue for some large quadrature computations that have recently been attempted on highly parallel computers.

Also, whereas one can readily compute very highprecision values with these methods, mathematicians often require "certificates," in other words rigorous guarantees that the approximation error cannot exceed a given level. For both of these reasons, we seek much more accurate and rigorous, yet readily computable error bounds for this class of quadrature methods. We present here some results in this direction. Due to space limitations, we have omitted many details and proofs. Full details may be found in [2].

1. Quadrature and the Euler-Maclaurin Formula. Atkinson's version of the Euler-Maclaurin formula is as follows [1, pg 285]: Let $m>0$ be an integer, and, for notational convenience throughout this paper assume that $h$ evenly divides $a$ and $b$ (in general it is only necessary that $h$ divide $b-a$ ). Further assume that the function $f(x)$ is at least $(2 m+2)$-times 
continuously differentiable on $[a, b]$. Then

$$
\begin{aligned}
& \int_{a}^{b} f(x) d x=h \sum_{j=a / h}^{b / h} f(j h)-\frac{h}{2}[f(a)+f(b)] \\
& -\sum_{i=1}^{m} \frac{h^{2 i} B_{2 i}}{(2 i) !}\left[D^{2 i-1} f(b)-D^{2 i-1} f(a)\right]+E(h, m),
\end{aligned}
$$

where $B_{j}$ denotes the $j$-th Bernoulli number, $D$ denotes the differentiation operator, and the error is given by [1, pg 288]:

$$
E(h, m)=\frac{(a-b) B_{2 m+2} D^{2 m+2} f(\xi)}{(2 m+2) !} h^{2 m+2},
$$

where $\xi \in(a, b)$.

When the function $f(t)$ and all of its derivatives are zero at the endpoints $a$ and $b$ (as with a smooth, bellshaped function), note that the second and third terms of the Euler-Maclaurin formula are zero. Thus, for such functions, the error of a simple step-function approximation to the integral, with interval $h$, is simply $E(h, m)$. But since, for any $m, E(h, m)$ is less than a constant (independent of $h$ ) times $h^{2 m+2}$, we conclude that the error goes to zero more rapidly than any fixed power of $h$.

This principle leads to state-of-the-art numerical integration schemes schemes, on transforming the integral of $F(x)$ on $[-1,1]$ to an integral of $f(t)=$ $F(g(t)) g^{\prime}(t)$ on $(-\infty, \infty)$, via the change of variable $x=g(t)$. Here $g(x)$ is any monotonic, infinitelydifferentiable function with the property that $g(x) \rightarrow$ \pm 1 as $x \rightarrow \pm \infty$, respectively, that also has the property that $g^{\prime}(x)$ and all higher derivatives rapidly approach zero for large positive and negative arguments. In this case we can write, for $h>0$,

$$
\begin{aligned}
\int_{-1}^{1} F(x) d x & =\int_{-\infty}^{\infty} F(g(t)) g^{\prime}(t) d t \\
& =h \sum_{j=-\infty}^{\infty} w_{j} F\left(x_{j}\right)+E(h)
\end{aligned}
$$

where $x_{j}=g(h j)$ and $w_{j}=g^{\prime}(h j)$. If $g^{\prime}(t)$ and its derivatives tend to zero sufficiently rapidly for large $t$, positive and negative, then-even in cases where

\begin{tabular}{|c|c|c|c|c|c|c|}
\hline$h$ & \multirow{2}{*}{\multicolumn{2}{|c|}{$\frac{\mathbf{e 2}}{10^{-2}}$}} & e4 & \multicolumn{2}{|c|}{ e6 } & e8 \\
\hline 1 & & & $10^{-5}$ & 10 & & $10^{-}$ \\
\hline $1 / 2$ & \multicolumn{2}{|c|}{$10^{-6}$} & $10^{-6}$ & \multicolumn{2}{|c|}{$10^{-8}$} & $10^{-10}$ \\
\hline $1 / 4$ & \multicolumn{2}{|c|}{$10^{-13}$} & $10^{-12}$ & \multicolumn{2}{|c|}{$10^{-17}$} & $10^{-2}$ \\
\hline $1 / 8$ & \multicolumn{2}{|c|}{$10^{-26}$} & $10^{-25}$ & \multicolumn{2}{|c|}{$10^{-34}$} & $10^{-4}$ \\
\hline $1 / 16$ & \multicolumn{2}{|c|}{$10^{-52}$} & $10^{-51}$ & \multicolumn{2}{|c|}{$10^{-68}$} & $10^{-}$ \\
\hline $1 / 32$ & \multicolumn{2}{|c|}{$10^{-104}$} & $10^{-102}$ & \multicolumn{2}{|c|}{$10^{-134}$} & $10^{-1}$ \\
\hline $1 / 64$ & \multicolumn{2}{|c|}{$10^{-206}$} & $10^{-204}$ & \multicolumn{2}{|c|}{$10^{-266}$} & $10^{-348}$ \\
\hline $1 / 128$ & \multicolumn{2}{|c|}{$10^{-411}$} & $10^{-409}$ & \multicolumn{2}{|c|}{$10^{-529}$} & $10^{-696}$ \\
\hline $1 / 256$ & \multicolumn{2}{|c|}{$10^{-821}$} & $10^{-81}$ & \multicolumn{2}{|c|}{$10^{-1056}$} & $10^{-139}$ \\
\hline & "h & & \begin{tabular}{l|l|} 
e10 \\
\end{tabular} & \begin{tabular}{l|l|} 
e12 \\
\end{tabular} & & \begin{tabular}{l|l} 
e14 \\
\end{tabular} \\
\hline & 1 & & $10^{-3}$ & $10^{-1}$ & & $0^{-1}$ \\
\hline & $1 / 2$ & & $10^{-8}$ & $10^{-3}$ & & $0^{-2}$ \\
\hline & $1 / 4$ & & $0^{-16}$ & $10^{-6}$ & & $0^{-3}$ \\
\hline & $1 / 8$ & & $0^{-33}$ & $10^{-11}$ & & $0^{-5}$ \\
\hline & $1 / 16$ & & $0^{-66}$ & $10^{-20}$ & & -10 \\
\hline & $1 / 32$ & & -132 & $10^{-37}$ & & -19 \\
\hline & $1 / 64$ & & -264 & $10^{-70}$ & & -37 \\
\hline & /128 & & -527 & $10^{-132}$ & & )$^{-68}$ \\
\hline & $/ 256$ & $10^{-}$ & -1053 & $10^{-249}$ & $10^{-}$ & -128 \\
\hline
\end{tabular}
$F(x)$ has an infinite derivative or an integrable singularity at one or both endpoints - the resulting integrand $f(t)=F(g(t)) g^{\prime}(t)$ will be a smooth bellshaped function for which the prior Euler-Maclaurin
Table 1. 'QUADERF' errors at successive values of $h$

argument applies. Thus, in such cases, the error $E(h)$ here decreases very rapidly as $h$ is reduced.

There are various such functions $g$ that work well in practice. Using $g(t):=\tanh t$ gives rise to tanh quadrature. Using $g(t):=\operatorname{erf}(t)$ gives rise to "error function" or erf quadrature. Using $g(t):=\tanh (\pi / 2$. $\sinh t)$ or $g(t):=\tanh (\sinh t)$ gives rise to tanh-sinh quadrature [9]. For integrand functions to be integrated on $(-\infty, \infty)$, so that a transformation from a finite interval to the entire real line is unnecessary, one can use $g(t):=\sinh t, g(t):=\sinh (\pi / 2 \cdot \sinh t)$ or $g(t):=\sinh (\sinh t)$.

One phenomenon that readily becomes apparent with these schemes is "quadratic convergence," where the number of correct digits is approximately doubled when $h$ is halved. This is given theoretical backing for "tanh" in [7] and for "tanh-sinh" in [6]. Table 1 shows this phenomenon for the following subset of the test problems used in a previous study with $g(t)=\operatorname{erf}(t)$ [4]. 


$$
\begin{array}{rll}
\text { e2 } & : & \int_{0}^{1} t^{2} \arctan t d t=(\pi-2+2 \log 2) / 12 \\
\text { e4 } & : & \int_{0}^{1} \frac{\arctan \left(\sqrt{2+t^{2}}\right)}{\left(1+t^{2}\right) \sqrt{2+t^{2}}} d t=5 \pi^{2} / 96 \\
\text { e6 }: & \int_{0}^{1} \sqrt{1-t^{2}} d t=\pi / 4 \\
\text { e8 }: & \int_{0}^{1} \log t^{2} d t=2 \\
\text { e10 }: & \int_{0}^{\pi / 2} \sqrt{\tan t} d t=\pi \sqrt{2} / 2 \\
\text { e12 }: & \int_{0}^{\infty} \frac{e^{-t}}{\sqrt{t}} d t=\sqrt{\pi} \\
\text { e14 }: & \int_{0}^{\infty} e^{-t} \cos t d t=1 / 2 .
\end{array}
$$

\section{Estimates of the Error Term.}

One estimate of the error term is given in $[10, \mathrm{pg}$ $34]$ and [8,pg. 211]: If the $2 \pi$-periodic function $f(z)$ is analytic in a strip $|\operatorname{Im}(z)|<c$, then the error in a trapezoidal (or step function) approximation to the integral is bounded by

$$
E(h) \leq \frac{4 \pi M}{e^{c N}-1},
$$

where $N$ is the number of evaluation points, $h=$ $2 \pi / N$, and $M$ is a bound on the absolute value of the function on the complex strip. This is an interesting result in that it begins to explain the phenomenon of quadratic convergence. However, it is not very useful in practice, because it requires one to locate singularities of a function in the complex plane and to find a maximum of the function on a complex strip.

What's more, the resulting estimate is not particularly accurate. For example, consider the function $f(t)=1 /\left(1+t^{2}\right)$, which on the interval $[-1,1]$ integrates to $\pi / 2$. When transformed with $x=$ $\tanh \left(4 \sinh t\right.$ ) (so that, to within a tolerance of $10^{-35}$, the function and its first few derivatives are zero at the endpoints of $[-\pi, \pi]$ ), the resulting function has a pole near $0.19763359 i$. Taking $c=0.197, M=$ 790, $N=64$ and $h=2 \pi / 64$ in (1), we obtain the estimate $3.32 \times 10^{-2}$. By contrast, the inexpensive error estimate that we introduce below (3), with $m=1$, gives $2.01832 \times 10^{-5}$ for the same $h$. The actual error in a trapezoidal approximation to the integral for this $h$, to ten significant digits, is $2.0183003673 \times 10^{-5}$.
To derive more accurate error bounds, we need to better understand the error term in the Euler-Maclaurin formula. To that end, we state two alternate forms of the error term, which were established in [2].

Theorem 1 The error term of the Euler-Maclaurin formula is given by:

$$
\begin{aligned}
& E(h, m)=2(-1)^{m-1}\left(\frac{h}{2 \pi}\right)^{2 m} \times \\
& \sum_{k=1}^{\infty} \frac{1}{k^{2 m}} \int_{a}^{b} \cos [2 k \pi(t-a) / h] D^{2 m} f(t) d t .
\end{aligned}
$$

For many integrand functions of interest, even the first term of the infinite summation here is an excellent approximation to the error. In other words, we can consider the approximation

$$
\begin{array}{r}
E_{1}(h, m)=2(-1)^{m-1}\left(\frac{h}{2 \pi}\right)^{2 m} \times \\
\int_{a}^{b} \cos [2 \pi(t-a) / h] D^{2 m} f(t) d t .
\end{array}
$$

We shall also introduce a second approximation, which we first discovered because of a 'bug' in our computer program:

Theorem 2 Suppose that $f(t)$ is defined on $[a, b]$, and has the property that $f(a)=f(b)=0$. Suppose further that the $f$ is at least $2 m$-times continuously differentiable on $[a, b]$, with $D^{k} f(a)=D^{k} f(b)=0$ for $1 \leq k \leq 2 \mathrm{~m}$. Also, as before assume that $h$ divides a and $b$. Further let $n \geq 1$ be any integer such that these conditions are also met with $m+n$ replacing $m$. Then

$$
\begin{gathered}
E(h, m)=h(-1)^{m-1}\left(\frac{h}{2 \pi}\right)^{2 m} \sum_{j=a / h}^{b / h} D^{2 m} f(j h) \\
+2(-1)^{n-1}\left(\frac{h}{2 \pi}\right)^{2 m+2 n} \sum_{k=1}^{\infty}\left(\frac{1}{k^{2 n}}+\frac{(-1)^{m}}{k^{2 m+2 n}}\right) \times \\
\int_{a}^{b} \cos [2 k \pi(t-a) / h] D^{2 m+2 n} f(t) d t .
\end{gathered}
$$

Theorem 2 immediately suggests the simple approximation

$E_{2}(h, m)=h(-1)^{m-1}\left(\frac{h}{2 \pi}\right)^{2 m} \sum_{j=a / h}^{b / h} D^{2 m} f(j h)$. 
We will make use $E_{2}$ in the computer implementations described in the next section.

One other estimate is worth mentioning.

Corollary 1 Under the hypotheses of Theorem 1 one has

$$
\begin{aligned}
&\left|E(h, m)-E_{1}(h, m)\right| \leq \\
& 2(\zeta(2 m)-1)\left(\frac{h}{2 \pi}\right)^{2 m} \int_{a}^{b}\left|D^{2 m} f(t)\right| d t .
\end{aligned}
$$

This bound can be used, for instance, to establish a rigorous "certificate" of the estimate $E_{1}(h, m)$, and thus (after computation of $E_{1}(h, m)$ ) of the quadrature result itself. Other useful bounds can also be derived. In particular, we can mirror Corollary 1 for Theorem 2:

Corollary 2 Under the hypotheses of Theorem 2 with $n=1$, one obtains

$$
\begin{aligned}
\left|E(h, m)-E_{2}(h, m)\right| \leq \\
2\left[\zeta(2 m)+(-1)^{m} \zeta(2 m+2)\right] \times \\
\left(\frac{h}{2 \pi}\right)^{2 m} \int_{a}^{b}\left|D^{2 m} f(t)\right| d t .
\end{aligned}
$$

This highlights what is gained by using $E_{2}(h, m)$ rather than $E_{1}(h, m)$ (note that (4) is particularly advantageous when $m$ is odd). In each of Corollary 1 and Corollary 2, by using the CauchySchwarz inequality, we may replace $\int_{a}^{b}\left|D^{2 m} f(t)\right| d t$ by $h \sqrt{\int_{a}^{b}\left|D^{2 m} f(t)\right|^{2} d t}$, which is often significantly smaller.

3. Implementations and Tests. We have implemented the error estimation formula (2) for $E_{1}(h, m)$, which requires integrations with the cosine terms, using Mathematica. We have also tried numerical integrations, using the computer programs described in [4], where it is important to use a much smaller integration interval than the $h$ that appears in the formula (we typically use $h / 8$ or $h / 16$ ). Implementing the formula (3) for $E_{2}(h, m)$ is even easier, and requires no symbolic manipulation except for finding the derivatives of the input function $F(t)$. Evaluation of $E_{2}(h, 1)$, for instance, can be done as follows: Let $g(t)$ be the function defining the quadrature scheme to be used. Then we can write

$$
\begin{aligned}
D^{2} f(t)= & \left.D^{2}[F(g(t)) D g(t)]\right] \\
= & F(g(t)) g^{\prime \prime \prime}(t)+F^{\prime}(g(t))\left[3 g^{\prime}(t) g^{\prime \prime}(t)\right] \\
& +F^{\prime \prime}(g(t))\left[g^{\prime}(t)\right]^{3}
\end{aligned}
$$

So for instance when $g(t)=\tanh t$,

$$
\begin{aligned}
D^{2} f(t)= & F(\tanh t)\left(-2 \operatorname{sech}^{4} t+4 \operatorname{sech}^{2} t \tanh ^{2} t\right) \\
& +F^{\prime}(\tanh t)\left(-6 \operatorname{sech}^{4} t \tanh t\right) \\
& +F^{\prime \prime}(\tanh t) \operatorname{sech}^{6} t
\end{aligned}
$$

Note that in formula (3) for $E_{2}(h, m)$, the function $D^{2 m} f(t)$ is evaluated at the same equi-spaced abscissas that are used in the quadrature calculation itself. It is often not necessary to perform the estimation calculations to the full precision used in the quadrature calculation (although in his paper we use full precision). In any event, these estimates can be computed quite rapidly.

Tables 2 through 5 include computational analysis of $E_{2}(h, m)$, using test functions

$$
\begin{array}{ll}
\mathbf{f 1}: & F_{1}(t)=1 /\left(1+t^{2}+t^{4}+t^{6}\right) \\
\mathbf{f 2}: & F_{2}(t)=\left(1-t^{4}\right)^{1 / 2} \\
\mathbf{f 3}: & F_{3}(t)=\left(1-t^{2}\right)^{-1 / 2} \\
\mathbf{f 4}: & F_{4}(t)=(1+t)^{2} \sin (2 \pi /(1+t)),
\end{array}
$$

with interval of integration. $[-1,1]$. The tanh-sinh rule was used for quadrature. In problems $\mathrm{f} 1, \mathrm{f} 2$ and f4, 400-digit arithmetic was employed. In problem f3, 1100-digit arithmetic was used, although 550-digit arithmetic suffices here if one employs a "secondary epsilon" technique described in [4]. Note that $F_{2}(t)$ has an infinite derivative at the endpoints, and $F_{3}(t)$ has a blow-up singularity at the endpoints, while $F_{4}(t)$ represents a worst case for these methods, since it is highly oscillatory near -1 . In particular, while the first two derivatives of the transformed function $f_{4}(t)$ tend to zero with large positive and negative arguments, the third and higher derivatives do not. (See Figure 1.)

Recall that for the tanh-sinh scheme, a function $F(t)$ to be integrated on $[-1,1]$ is transformed to the function $f(t)=F(\tanh (\sinh t)) \operatorname{sech}^{2}(\sinh t) \cosh t$, integrated over $(-\infty, \infty)$. For the problems $\mathrm{f} 1, \mathrm{f} 2$ and $\mathrm{f} 4$, the "infinite" integration interval $[a, b]$ is taken to be $[-7,7]$, which is (provably) sufficient to insure that all 


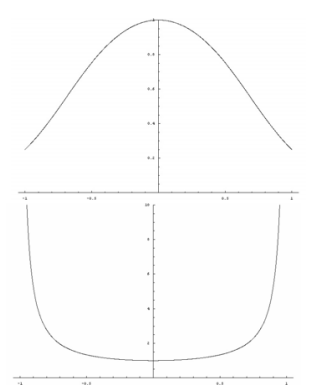

Figure 1. The four test functions $F_{1}, F_{2}, F_{3}, F_{4}$

of these computations agree with values on $(-\infty, \infty)$ to within $10^{-400}$ in each case. For the problem $\mathrm{f3}$, the interval is $[-8,8]$, which is sufficient to insure accuracy to within $10^{-600}$.

The values $E(h)$ shown in the first column of the four tables are the actual errors of the quadrature results, namely $E(h)=\int_{-1}^{1} F(t) d t-Q(h)$, where $Q(h)$ is the numerical approximation to the integral using the tanh-sinh rule. The correct quadrature results for the first three problems are $\pi / 4+$ $\log (1+\sqrt{2}) / \sqrt{2}, \sqrt{\pi} \Gamma(5 / 4) / \Gamma(7 / 4)$ and $\pi$, respectively. We know of no classical closed-form evaluation for problem $\mathrm{f} 4$, but in terms of the cosine integral the result equals $-\frac{4}{15} \pi^{5} \mathrm{Ci}(\pi)+\frac{4}{15} \pi^{3}-\frac{8}{5} \pi \approx$ $-2.76989612386024129018 \ldots$ Here $\mathrm{Ci}(x)=\gamma+$ $\ln (x)+\int_{0}^{x} \frac{\cos (t)-1}{t} d t$, and $\gamma$ is Euler's constant.

Except for problem f4, we include results for $E_{2}(h, m)$ with $m=1,2,3,4$, or, in other words, estimates based on the second, fourth, sixth and eighth derivatives of $f(t)$, respectively.

It is clear from these results that the estimates $E_{2}(h, m)$ are extremely accurate in the first three test problems. In each of these problems, $E_{2}(h, 1)$ through $E_{2}(h, 4)$ all agree with the actual errors to at least eight significant digits when $h=1 / 4$, and to more than 120 significant digits when $h=1 / 64$ (in the problem $\mathrm{f} 3$, the four estimates are correct to 270 significant digits when $h=1 / 64)$. Indeed, as $h$ is reduced the estimates actually increase in relative precision as well as in absolute precision, approximately doubling in relative precision (in digits) with each halving of the interval $h$ (in other words, exhibiting quadratic convergence). The estimate $E_{2}(h, 1)$ is always the most ac- curate, although the higher-level estimates are never far behind.

For the pathological problem f4, with highly oscillatory behavior near -1 , the estimates are not nearly as accurate. But even here they are within a factor of three for all $h \leq 1 / 8$-more than sufficient to determine the order of magnitude of error in practical computation.

4. A Mathematical Physics Example. One of the present authors (Borwein) and David Broadhurst found the following conjectured identity:

$$
\begin{aligned}
I & =\frac{24}{7 \sqrt{7}} \int_{\pi / 3}^{\pi / 2} \log \left|\frac{\tan t+\sqrt{7}}{\tan t-\sqrt{7}}\right| d t \stackrel{?}{=} L_{-7}(2) \\
& =\sum_{n=0}^{\infty}\left[\frac{1}{(7 n+1)^{2}}+\frac{1}{(7 n+2)^{2}}-\frac{1}{(7 n+3)^{2}}\right. \\
& \left.+\frac{1}{(7 n+4)^{2}}-\frac{1}{(7 n+5)^{2}}-\frac{1}{(7 n+6)^{2}}\right]
\end{aligned}
$$

This integral arose out of studies in quantum field theory, in analysis of the volume of ideal tetrahedra in hyperbolic space. Such studies are currently of substantial interest to mathematical physicists, topologists and knot theorists. Note that the integrand function has a nasty singularity at $t=\arctan (\sqrt{7})$. The '?' indicates no formal proof is yet known. In [3], we applied a highly parallel implementation of the tanhsinh quadrature algorithm to calculate the numerical value of this integral to 20,000-digit precision, and found that it agreed precisely with a 20,000-digit evaluation of the right-hand side. Our integral evaluation employed only an ad-hoc error estimation procedure, and colleagues challenged us to find a more modest but rigorous "certificate" for this result. To that end, we note that (5) can be written as

$$
\begin{aligned}
I= & \frac{24}{7 \sqrt{7}}\left(b_{1} \int_{-1}^{1} w_{1}(t) d t-b_{2} \int_{-1}^{1} w_{2}(t) d t\right. \\
& \left.+b_{3} \int_{-1}^{1} w_{3}(t) d t-b_{4} \int_{-1}^{1} w_{4}(t) d t\right)
\end{aligned}
$$

where

$$
\begin{aligned}
& w_{1}(t)=\log \left[\tan \left(a_{1}+b_{1} t\right)+c_{1}\right], \\
& w_{2}(t)=\log \left[-\tan \left(a_{2}+b_{2} t\right)+c_{2}\right], \\
& w_{3}(t)=\log \left[\tan \left(a_{3}+b_{3} t\right)+c_{3}\right], \\
& w_{4}(t)=\log \left[\tan \left(a_{4}+b_{3} t\right)+c_{4}\right]
\end{aligned}
$$


and where

$$
\begin{aligned}
& a_{1}=(\arctan (\sqrt{7})+\pi / 3) / 2, \\
& b_{1}=(\arctan (\sqrt{7})-\pi / 3) / 2, \\
& c_{1}=\sqrt{7} \\
& a_{2}=(\arctan (\sqrt{7})+\pi / 3) / 2, \\
& b_{2}=(\arctan (\sqrt{7})-\pi / 3) / 2, \\
& c_{2}=\sqrt{7}, \\
& a_{3}=(\arctan (\sqrt{7})+\pi / 2) / 2, \\
& b_{3}=(-\arctan (\sqrt{7})+\pi / 2) / 2, \\
& c_{3}=\sqrt{7}, \\
& a_{4}=(\arctan (\sqrt{7})+\pi / 2) / 2, \\
& b_{4}=(-\arctan (\sqrt{7})-\pi / 2) / 2, \\
& c_{4}=-\sqrt{7} .
\end{aligned}
$$

We calculated the estimated errors $E_{1}(h, 1)$ through $E_{1}(h, 5)$, for each of the four component integrals, using the tanh-sinh rule, with $h=10^{-4}$ and 220digit arithmetic. We found that each of these $20 \mathrm{er}-$ rors is less than $10^{-200}$ (in fact, this is true even when $h=10^{-2}$ ). As part of the computation of the error bound $E_{2}(h, 5)$, we determined that $\left|D^{10} f(t)\right|$, where $f(t)=w_{4}(g(t)) g^{\prime}(t)$, is bounded by $10^{6}$ within the interval $[-3,3]$, and has relatively negligible values outside this interval. This fact was confirmed by symbolic computation using Mathematica. Applying formula (4) and using the note immediately following (4) on a hand calculator, we obtain a bound of $3.82 \times 10^{-49}$ for this integral. The other three integrals yield slightly smaller bounds. Hence, $3.82 \times 10^{-49}$ is a certificate for the integral (5).

Of course, this is all premised on: (1) correct implementations of the algorithms, (2) faithful implementation of the underlying arithmetic (performed using the ARPREC software), (3) correct symbolic differentiation (performed using Mathematica), and (4) correct location of the maximum of the tenth-order derivatives (performed using ARPREC and confirmed using Mathematica).

5. Conclusion. We have derived two estimates of the error in Euler-Maclaurin-based quadrature, one of which is particularly simple to implement, since it only involves summation of derivatives of the transformed function, at the same abscissas as the quadrature calculation itself. It appears, from our results in several test problems, that the simplest instance of these estimates, namely $E_{2}(h, 1)$, is not only adequate, but in fact very accurate once $h$ is even modestly small. What is more, the difference between this estimate and the actual error can be bounded with an easily computed formula, thus permitting "certificates" of quadrature values computed using Euler-Maclaurin-based schemes.

Acknowledgements. Bailey's work supported by the Director, Office of Computational and Technology Research, Division of Mathematical, Information, and Computational Sciences of the U.S. Department of Energy, under contract number DE-AC02-05CH11231. Borwein's work supported in part by NSERC and the Canada Research Chair Programme.

\section{References}

[1] K. E. Atkinson. Elementary Numerical Analysis. John Wiley and Sons, New York, 1993.

[2] D. H. Bailey and J. M. Borwein. Effective bounds in euler-maclaurin-based quadrature. http: //crd.lbl.gov/ dhbailey/dhbpapers/ em-error.pdf, 2005.

[3] D. H. Bailey and J. M. Borwein. Highly parallel, high-precision numerical integration. http: //crd.1bl.gov/ dhbailey/dhbpapers / quadparallel.pdf, 2005.

[4] D. H. Bailey and X. S. Li. A comparison of three high-precision quadrature schemes. Experimental Mathematics, 14(3):317-329, 2005.

[5] J. M. Borwein and D. H. Bailey. Mathematics by Experiment. A.K. Peters, Natick MA, 2004.

[6] J. M. Borwein and P. L. Ye. Quadratic convergence of tanh-sinh quadrature rule. http://www.cs.dal.ca/ jborwein/ tanhsinh.pdf, 2006.

[7] S. Haber. The tanh rule for numerical integration. SIAM Journal on Numerical Analysis, 14:668-685, 1977.

[8] R. Kress. Numerical Analysis. Springer Graduate Texts in Mathematics, New York.

[9] H. Takahasi and M. Mori. Double exponential formulas for numerical integration. Publications of RIMS, 9:721-741, 1974.

[10] J. A. C. Weideman. Numerical integration of periodic functions: A few examples. American Mathematical Monthly, 109(1):21-36, Jan 2002. 


\begin{tabular}{|r|r|r|r|r|r|}
\hline$h$ & $E(h)$ & $\left|E(h)-E_{2}(h, 1)\right|$ & $\left|E(h)-E_{2}(h, 2)\right|$ & $\left|E(h)-E_{2}(h, 3)\right|$ & $\left|E(h)-E_{2}(h, 4)\right|$ \\
\hline $1 / 1$ & $5.34967 \times 10^{-3}$ & $9.81980 \times 10^{-4}$ & $4.77454 \times 10^{-3}$ & $1.87712 \times 10^{-2}$ & $6.48879 \times 10^{-2}$ \\
$1 / 2$ & $-3.36641 \times 10^{-4}$ & $1.12000 \times 10^{-7}$ & $5.60084 \times 10^{-7}$ & $2.35316 \times 10^{-6}$ & $9.53208 \times 10^{-6}$ \\
$1 / 4$ & $-3.73280 \times 10^{-8}$ & $1.67517 \times 10^{-16}$ & $8.37583 \times 10^{-16}$ & $3.51785 \times 10^{-15}$ & $1.42389 \times 10^{-14}$ \\
$1 / 8$ & $5.58389 \times 10^{-17}$ & $2.29357 \times 10^{-32}$ & $1.14679 \times 10^{-31}$ & $4.81651 \times 10^{-31}$ & $1.94954 \times 10^{-30}$ \\
$1 / 16$ & $-7.64525 \times 10^{-33}$ & $2.07256 \times 10^{-64}$ & $1.03628 \times 10^{-63}$ & $4.35237 \times 10^{-63}$ & $1.76167 \times 10^{-62}$ \\
$1 / 32$ & $-6.90852 \times 10^{-65}$ & $7.23441 \times 10^{-129}$ & $3.61721 \times 10^{-128}$ & $1.51923 \times 10^{-127}$ & $6.14925 \times 10^{-127}$ \\
$1 / 64$ & $-2.41147 \times 10^{-129}$ & $9.08805 \times 10^{-259}$ & $4.54403 \times 10^{-258}$ & $1.90849 \times 10^{-257}$ & $7.72485 \times 10^{-257}$ \\
\hline
\end{tabular}

Table 2. Results for $F_{1}(t)=1 /\left(1+t^{2}+t^{4}+t^{6}\right)$

on $[-1,1]$.

\begin{tabular}{|r|r|r|r|r|r|}
\hline$h$ & $E(h)$ & $\left|E(h)-E_{2}(h, 1)\right|$ & $\left|E(h)-E_{2}(h, 2)\right|$ & $\left|E(h)-E_{2}(h, 3)\right|$ & $\left|E(h)-E_{2}(h, 4)\right|$ \\
\hline $1 / 1$ & $2.92136 \times 10^{-2}$ & $4.12347 \times 10^{-5}$ & $2.06449 \times 10^{-4}$ & $8.69796 \times 10^{-4}$ & $3.54584 \times 10^{-3}$ \\
$1 / 2$ & $1.37266 \times 10^{-5}$ & $3.40342 \times 10^{-11}$ & $1.70174 \times 10^{-10}$ & $7.14758 \times 10^{-10}$ & $2.89332 \times 10^{-9}$ \\
$1 / 4$ & $1.13445 \times 10^{-11}$ & $1.60476 \times 10^{-21}$ & $8.02380 \times 10^{-21}$ & $3.36999 \times 10^{-20}$ & $1.36405 \times 10^{-19}$ \\
$1 / 8$ & $5.34920 \times 10^{-22}$ & $1.06920 \times 10^{-41}$ & $5.34599 \times 10^{-41}$ & $2.24532 \times 10^{-40}$ & $9.08818 \times 10^{-40}$ \\
$1 / 16$ & $3.56399 \times 10^{-42}$ & $1.36460 \times 10^{-81}$ & $6.82298 \times 10^{-81}$ & $2.86565 \times 10^{-80}$ & $1.15991 \times 10^{-79}$ \\
$1 / 32$ & $4.54865 \times 10^{-82}$ & $6.34476 \times 10^{-161}$ & $3.17238 \times 10^{-160}$ & $1.33240 \times 10^{-159}$ & $5.39305 \times 10^{-159}$ \\
$1 / 64$ & $2.11492 \times 10^{-161}$ & $3.89818 \times 10^{-319}$ & $1.94909 \times 10^{-318}$ & $8.18618 \times 10^{-318}$ & $3.31345 \times 10^{-317}$ \\
\hline
\end{tabular}

Table 3. Results for $F_{2}(t)=\left(1-t^{4}\right)^{1 / 2}$ on

$[-1,1]$.

\begin{tabular}{|r|r|r|r|r|r|}
\hline$h$ & $E(h)$ & $\left|E(h)-E_{2}(h, 1)\right|$ & $\left|E(h)-E_{2}(h, 2)\right|$ & $\left|E(h)-E_{2}(h, 3)\right|$ & $\left|E(h)-E_{2}(h, 4)\right|$ \\
\hline $1 / 1$ & $-9.38039 \times 10^{-5}$ & $2.00740 \times 10^{-7}$ & $1.00302 \times 10^{-6}$ & $4.20595 \times 10^{-6}$ & $1.69621 \times 10^{-5}$ \\
$1 / 2$ & $6.69591 \times 10^{-8}$ & $1.17622 \times 10^{-15}$ & $5.88109 \times 10^{-15}$ & $2.47006 \times 10^{-14}$ & $9.99785 \times 10^{-14}$ \\
$1 / 4$ & $-3.92072 \times 10^{-16}$ & $2.48852 \times 10^{-32}$ & $1.24426 \times 10^{-31}$ & $5.22589 \times 10^{-31}$ & $2.11524 \times 10^{-30}$ \\
$1 / 8$ & $-8.29506 \times 10^{-33}$ & $2.17847 \times 10^{-66}$ & $1.08924 \times 10^{-65}$ & $4.57479 \times 10^{-65}$ & $1.85170 \times 10^{-64}$ \\
$1 / 16$ & $-7.26158 \times 10^{-67}$ & $4.51319 \times 10^{-135}$ & $2.25659 \times 10^{-134}$ & $9.47769 \times 10^{-134}$ & $3.83621 \times 10^{-133}$ \\
$1 / 32$ & $-1.50440 \times 10^{-135}$ & $3.19951 \times 10^{-272}$ & $1.59976 \times 10^{-271}$ & $6.71897 \times 10^{-271}$ & $2.71958 \times 10^{-270}$ \\
$1 / 64$ & $1.06650 \times 10^{-272}$ & $4.25792 \times 10^{-546}$ & $2.12896 \times 10^{-545}$ & $8.94163 \times 10^{-545}$ & $3.61923 \times 10^{-544}$ \\
\hline
\end{tabular}

Table 4. Results for $F_{3}(t)=\left(1-t^{2}\right)^{-1 / 2}$ on $[-1,1]$.

\begin{tabular}{|r|r|r|}
\hline$h$ & $E(h)$ & $\left|E(h)-E_{2}(h, 1)\right|$ \\
\hline $1 / 1$ & $-6.45859 \times 10^{-1}$ & $3.54091 \times 10^{0}$ \\
$1 / 2$ & $2.54145 \times 10^{-2}$ & $7.23759 \times 10^{-1}$ \\
$1 / 4$ & $-1.69389 \times 10^{-2}$ & $1.00104 \times 10^{-1}$ \\
$1 / 8$ & $-8.84080 \times 10^{-3}$ & $1.37392 \times 10^{-2}$ \\
$1 / 16$ & $1.08078 \times 10^{-3}$ & $8.85166 \times 10^{-4}$ \\
$1 / 32$ & $-2.39628 \times 10^{-4}$ & $8.44565 \times 10^{-5}$ \\
$1 / 64$ & $-4.87134 \times 10^{-5}$ & $3.42934 \times 10^{-5}$ \\
\hline
\end{tabular}

Table 5. Results for $F_{4}(t)=(1+$ $t)^{2} \sin (2 \pi /(1+t))$ on $[-1,1]$. 